\title{
A Simple Theory of Mixtures for the Study of the Temperature Dependence of Thermo-structural Properties of Al-Ga Liquid Alloy
}

\author{
Kritika Khanal, Shashit Kumart Yadav and Devendra Adhikari*
}

Department of Physics, Mahendra Morang Adarsh Multiple Campus,

Tribhuvan University, Biratnagar, 56600 Nepal

*Corresponding author: adksbdev@yahoo.com, devendra.adhikari@mmamc.tu.edu.np

Published online: 25 November 2020

To cite this article: Khanal, K. Yadav, S. K. \& Adhikari, D. (2020). A simple theory of mixtures for the study of the temperature dependence of thermo-structural properties of Al-Ga liquid alloy. J. Phys. Sci., 31(3), 87-100. https://doi.org/10.21315/jps2020.31.3.7

To link to this article: https://doi.org/10.21315/jps2020.31.3.7

\begin{abstract}
A simple theory of mixtures for symmetric alloys was used to study the thermodynamic and structural properties of the Al-Ga alloy in the liquid state at $1023 \mathrm{~K}$. The computed thermodynamic properties are in a very good agreement with the observed values and show that the alloy is weakly interacting. The computed structural properties show that the Al-Ga alloy is segregating in nature which is in agreement with the observed values. The excess free energy of mixing, activity of monomers and concentration fluctuation in the long wavelength limit was extrapolated to higher temperatures by optimising the temperature dependent parameters. As the temperature increases, the behaviour of the alloy shifts towards the ideality.
\end{abstract}

Keywords: Simple theory of mixture, Al-Ga alloy, hetero-pairs, concentration fluctuation, diffusion coefficients

\section{INTRODUCTION}

Understanding the mixing behaviour of the constituents of an alloy in the liquid state is of great importance in developing the desired materials in the solid-state. The tendency to form hetero-pairs or homo-pairs in liquid alloys is also a matter of interest to researchers. The symmetric and asymmetric behaviours of an alloy also provide enormous information for further research. However, due to the state of disorder present in a liquid state, the formulation of an exact theoretical model for the liquid alloy to understand thermo-structural properties is a difficult task. To comprehend the complexities of liquid alloys, a number of models have, therefore, 
been employed by researchers. ${ }^{1-12}$ In the present work, a simple theory of mixture for symmetric alloy was employed to study the thermodynamic and structural properties of aluminium-gallium (Al-Ga) alloy at $1023 \mathrm{~K}$ in the liquid state. ${ }^{14,15}$

In the simple theory of mixtures, the heat of mixing is only related to the bond energies between adjacent atoms, and the interatomic distances and bond energies are independent of composition. The simple theory of mixture is applicable to a binary solution in which the atomic size factor of the constituents, described in the following formula, should be within $15 \%:^{16}$

$$
\text { Atomic size factor }=\left(\frac{r_{\text {solute }}-r_{\text {solvent }}}{r_{\text {solvent }}} \times 100\right)
$$

In the Al-Ga alloy, the atomic size factor is very small and is less than $15 \%$. It is a well-known fact that the atomic radius increases on moving downwards a group in the modern periodic table. Even though $\mathrm{Al}$ comes first and $\mathrm{Ga}$ in its next period, the atomic radius of $\mathrm{Ga}$ is less than that of $\mathrm{Al}$. This interesting and exceptional fact led the authors to investigate the Al-Ga alloy. Further, if there is a large difference in the electronegativity of the constituents of a solution, charge transfer occurs, and the formation of strong ionic bonding is likely to exist. In Al-Ga alloy, the electronegativity of $\mathrm{Al}(=1.61)$ and $\mathrm{Ga}(=1.81)$ are comparable to each other and hence, there is less chance of forming ionic bonding between them. For this reason, a simple theory of mixture was employed to explain the mixing behaviour of the Al-Ga alloy in the liquid state.

The outline of this paper is as follows. In Section 2, the theoretical basis of our work is presented. Section 3 gives the results and discussion of this work. Finally, the conclusions are outlined in Section 4.

\section{THEORETICAL BASIS}

Consider metal A (= Al) and metal $\mathrm{B}(=\mathrm{Ga})$ mixed in the molten state. The excess Gibb's free energy of mixing $\left(G_{M}^{X S}\right)$ of the binary mixture can be expressed in a polynomial in terms of concentration as: $:^{14,15}$

$$
G_{M}^{X S}=x_{1} x_{2}\left\{A_{0}+A_{1}\left(x_{1}-x_{2}\right)+A_{2}\left(x_{1}-x_{2}\right)^{2}+\ldots\right\}
$$

or

$$
G_{M}^{X S}=x(1-x)\left\{A_{0}+A_{1}(1-2 x)+A_{2}(1-2 x)^{2}+\ldots\right\}
$$


where the coefficients $A_{0}, A_{1}, A_{2}, \ldots$ are independent of composition but dependent on temperature $\mathrm{T}$ and on pressure $\mathrm{P}, x=x_{2}$ is the concentration of $\mathrm{B}$, and $1-x=x_{1}$ is the concentration $\mathrm{A}$. For a symmetrical binary mixture, the coefficient $A_{1}=0 .{ }^{17}$ Then, Equation 1 becomes:

$$
G_{M}^{X S}=x(1-x)\left\{A_{0}+A_{2}(1-2 x)^{2}\right\}
$$

The excess entropy of mixing for the symmetrical mixture can be obtained using a standard thermodynamic relation $S_{M}^{X S}=-\frac{\partial G_{M}^{X S}}{\partial T}$ as:

$$
S_{M}^{X S}=-x(1-x)\left\{\frac{\partial A_{0}}{\partial T}+\frac{\partial A_{2}}{\partial T}(1-2 x)^{2}\right\}
$$

The heat of mixing, Gibb's free energy of mixing and entropy of mixing are related by the relation:

$$
G_{M}^{X S}=H_{M}-T S_{M}^{X S}
$$

Using this relation together with Equations 2 and 3, we have:

$$
H_{M}=x(1-x)\left[\left\{A_{0}-T \frac{\partial A_{0}}{\partial T}\right\}+\left\{A_{2}-T \frac{\partial A_{2}}{\partial T}\right\}(1-2 x)^{2}\right]
$$

Now, the activity of monomers of the symmetrical binary mixture is given as: ${ }^{17}$

$$
\begin{aligned}
& R T \ln \left(a_{1} / x_{1}\right)=x_{2}^{2}\left[A_{0}+A_{2}\left(x_{2}-x_{1}\right)\left(x_{2}-5 x_{1}\right)\right] \\
& R T \ln \left(a_{2} / x_{2}\right)=x_{1}^{2}\left[A_{0}+A_{2}\left(x_{1}-x_{2}\right)\left(x_{1}-5 x_{2}\right)\right]
\end{aligned}
$$

where $a_{1}$ and $a_{2}$ are the activity coefficients of monomers A and B of the binary mixture, respectively. These activity coefficients can be related to the activities of monomers $\mathrm{A}$ and $\mathrm{B}$ by the respective relations $a_{1}=x_{1} \gamma_{1}$ and $a_{2}=x_{2} \gamma_{2}$.

The quantity that is important to understanding the ordering and segregating nature of a binary mixture, called concentration fluctuation in the long wavelength limit $\left(S_{C C}(0)\right)$, can be related to the Gibb's free energy of mixing as follows: ${ }^{2}$

$$
S_{C C}(0)=\frac{R T}{\left(\frac{\partial^{2} G M}{\partial x^{2}}\right)_{T, P, N}}
$$


Therefore, using Equations 2 and 6 with $G_{M}=G_{M}^{X S}+R T\left(x_{1} \ln x_{1}+x_{2} \ln x_{2}\right) S_{C C}(0)$ for a symmetrical binary mixture is given as:

$$
S_{C C}(0)=\frac{x(1-x)}{1-\frac{2 x(1-x) A_{0}}{R T}+\frac{2 x(1-x) A_{2}}{R T}+\left(24 x-24 x^{2}-5\right)}
$$

The $S_{C C}(0)$ can also be related to the activity of the monomers of the binary mixture, called the observed value, is given as: ${ }^{17}$

$$
S_{C C}(0)=x_{2} a_{1}\left(\frac{\partial a_{1}}{\partial x_{2}}\right)_{T, P, N}^{-1}=x_{1} a_{2}\left(\frac{\partial a_{2}}{\partial x_{1}}\right)_{T, P, N}^{-1}
$$

To know greater insight into the atomic ordering of a binary alloy, Warren-Cowley short range order parameter $\left(\alpha_{1}\right)$ can be determined. ${ }^{18,19}$ The expression for $\alpha_{1}$ is given as:

$$
\alpha_{1}=\frac{S-1}{(z-1) S+1}, \quad \frac{S_{C C}(0)}{S_{C C}^{i d}(0)}
$$

where $S_{C C}^{i d}(0)=x_{1} x_{2}$ and $z$ is the coordination number.

The segregating and ordering nature of an alloy can also be judged by means of the ratio of mutual and ideal diffusion coefficients. The mutual diffusion coefficients $\left(D_{M}\right)$ and ideal diffusion coefficient $\left(D_{i d}\right)$ are given as: ${ }^{20,21}$

$$
\begin{gathered}
D_{M}=D_{i d} \frac{\partial \ln a}{\partial x} \\
D_{i d}=x D_{1}+(1-x) D_{2}
\end{gathered}
$$

where $D_{1}$ and $D_{2}$ are the self-diffusion coefficients of pure components $\mathrm{A}$ and $\mathrm{B}$, respectively.

The mutual and ideal diffusion coefficients can be related to concentration fluctuation in the long wavelength limit as follows: ${ }^{20,21}$

$$
\frac{D_{M}}{D_{i d}}=\frac{x_{1} x_{2}}{S_{C C}(0)}=1-\frac{2 x(1-x) A_{0}}{R T}+\frac{2 x(1-x) A_{2}}{R T} 24 x-24 x^{2}-5
$$


The parameters $A_{0}$ and $A_{2}$ can be extrapolated to different temperatures using the relation

$$
\begin{aligned}
& A_{0}^{\prime}=A_{0}+\frac{\partial A_{0}}{\partial T} d T \\
& A_{2}^{\prime}=A_{2}+\frac{\partial A_{2}}{\partial T} d T
\end{aligned}
$$

where $d T$ is the difference in temperature.

\section{RESULTS AND DISCUSSION}

The parameters $A_{0}$ and $A_{2}$ for the Al-Ga alloy at $1023 \mathrm{~K}$ were estimated by the least square method using the observed values of the excess free energy of mixing and Equation 2 in the concentration range 0.1 to 0.9 of $\mathrm{Al}^{22}$ The respective best fit value of $A_{0}$ and $A_{2}$ were found to be $1254 \mathrm{~J} \mathrm{~mol}^{-1}$ and 0 at $1023 \mathrm{~K}$ for the Al-Ga alloy. The positive value of $A_{0}$ indicates that there is a tendency of phase segregation (preferred with like atom pairing) in the Al-Ga alloy in the molten state. The computed values of the $G_{M}^{X S}$ obtained from Equation 1 were plotted against $x$ (concentration of $\mathrm{Ga}$ ) for the Al-Ga alloy at $1023 \mathrm{~K}$ along with their observed values in Figure 1.

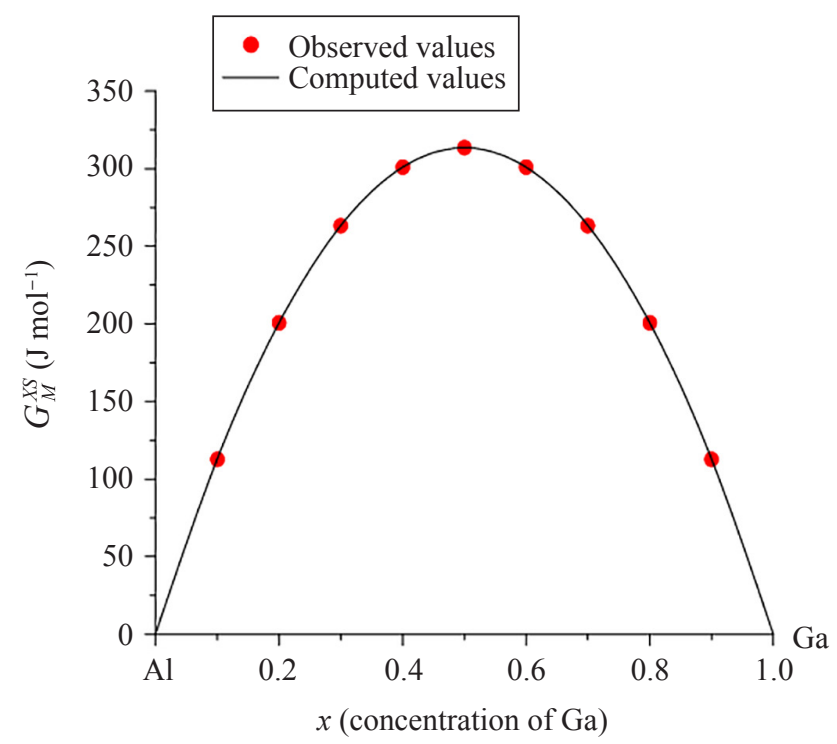

Figure 1: Excess free energy of mixing $G_{M}^{X S}$ vs. concentration of Ga of liquid Al-Ga alloy at $1023 \mathrm{~K}$. 
The computed and observed values of $G_{M}^{X S}$ for the Al-Ga alloy at $1023 \mathrm{~K}$ are in excellent agreement with one another at all concentrations. ${ }^{22}$ Both the computed and observed values showed that the Al-Ga alloy at $1023 \mathrm{~K}$ is a symmetric alloy with a maximum value at equiatomic composition. The computed maximum value of $G_{M}^{X S}\left(=313.5 \mathrm{~J} \mathrm{~mol}^{-1}\right)$ is exactly equal to the observed maximum value $\left(=313.5 \mathrm{~J} \mathrm{~mol}^{-1}\right)$ at equiatomic compositions. ${ }^{21}$ It is also worth mentioning that the maximum value of $G_{M}^{X S}$, as evident from the computed and observed values, implies that the $\mathrm{Al}-\mathrm{Ga}$ alloy in the molten state at $1023 \mathrm{~K}$ is a weakly interacting segregating alloy. The positive value of $G_{M}^{X S}$ at all compositions is also a confirmation of the phase segregating nature of Al-Ga alloy at $1023 \mathrm{~K}$.

Activity, a thermodynamic quantity that can be directly obtained from experiments, can be used to compute the thermodynamic functions, such as free energy of mixing and concentration fluctuation in the long wavelength limit. The deviations of a binary solution from an ideal behaviour can be understood by means of this quantity. The magnitudes of the activities of monomers of a solution depend on the bond energies between the constituents of the mixture. The activities of $\mathrm{Al}$ and $\mathrm{Ga}$ in the Al-Ga alloy at $1023 \mathrm{~K}$ were computed using Equation 5 and the estimated values of $A_{0}$ and $A_{2}$. The computed and observed values of the activities of $\mathrm{Al}$ and $\mathrm{Ga}$ are plotted in Figure 2. The computed and observed values of the activities of the monomers of the Al-Ga alloy at $1023 \mathrm{~K}$ were found to be in excellent agreement with one another. ${ }^{22}$ The activity coefficients of the monomers of the Al-Ga alloy at $1023 \mathrm{~K}$ were found to be greater than unity (even though by a small amount) for all compositions. This clearly indicates that the Al-Ga alloy at $1023 \mathrm{~K}$ exhibits a positive deviation from Rauolt's law. Therefore, in the case of Al-Ga alloy, less energy is required to separate the constituent atoms, i.e., the alloy is of a segregating nature.

In order to compute the entropy of mixing of the Al-Ga alloy at $1023 \mathrm{~K}$ in the liquid state, the temperature derivative of the energy parameter $\frac{\partial A_{0}}{\partial T}$ and $\frac{\partial A_{2}}{\partial T}$ were determined by the method of optimisation using the observed value of the excess entropy of mixing and Equation 3. The best fit values were $\frac{\partial A_{0}}{\partial T}=-1.071$ $\mathrm{J} \mathrm{mol}^{-1} \mathrm{~K}^{-1}$ and $\frac{\partial A_{2}}{\partial T}=-0.534 \mathrm{~J} \mathrm{~mol}^{-1} \mathrm{~K}^{-1}$. The computed and observed values of the entropy of mixing for the Al-Ga alloy at $1023 \mathrm{~K}$ were found to agree very well with one another (Figure 3 ). ${ }^{22}$ Both the observed and computed value showed that the entropy of mixing was symmetrical about equiatomic composition. The heat of mixing of the Al-Ga alloy at $1023 \mathrm{~K}$ was calculated using Equation 4 and the values of $A_{0}, A_{2}, \frac{\partial A_{0}}{\partial T}$ and $\frac{\partial A_{2}}{\partial T}$. The computed and observed values of heat of mixing were found to be a reasonable agreement with each other (Figure 4). 
Both the computed and observed values of the heats of mixing are positive at all compositions. The positive value of heat of mixing of the Al-Ga alloy is also indicative of the segregating nature of the alloy. However, the small positive value of the heat of mixing implies that the bonding strength between the like atoms is weak.

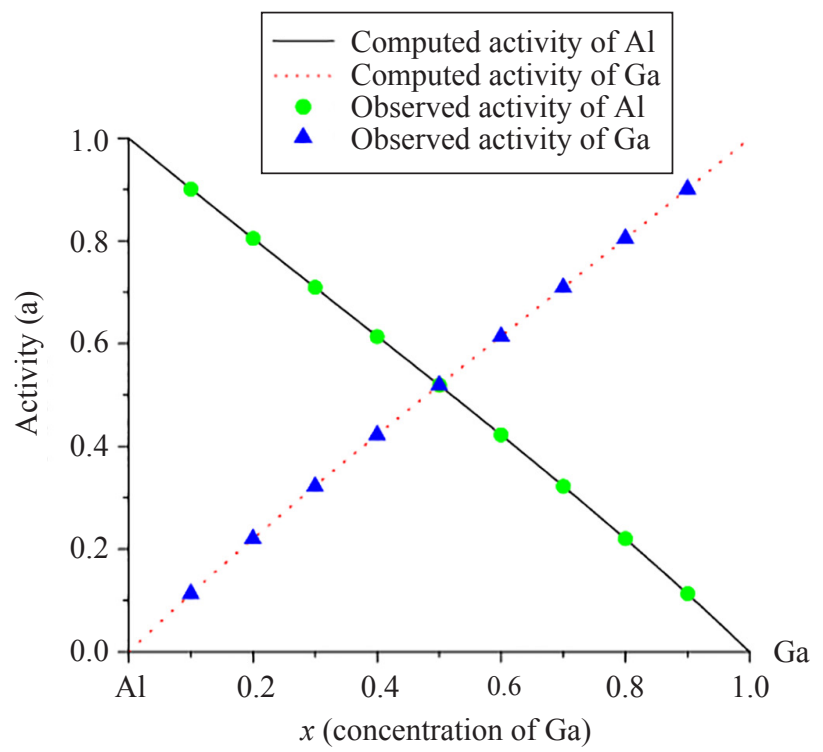

Figure 2: Activity of $\mathrm{Al}$ and Ga vs. concentration of Ga of liquid Al-Ga alloy at $1023 \mathrm{~K}$.

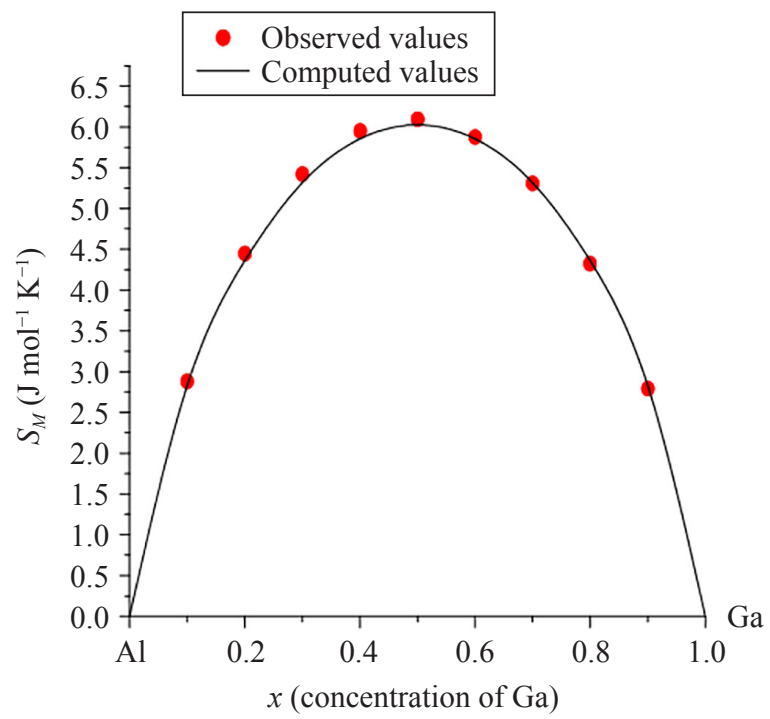

Figure 3: Entropy of mixing $S_{M}$ vs. concentration of Ga of liquid Al-Ga alloy at $1023 \mathrm{~K}$. 


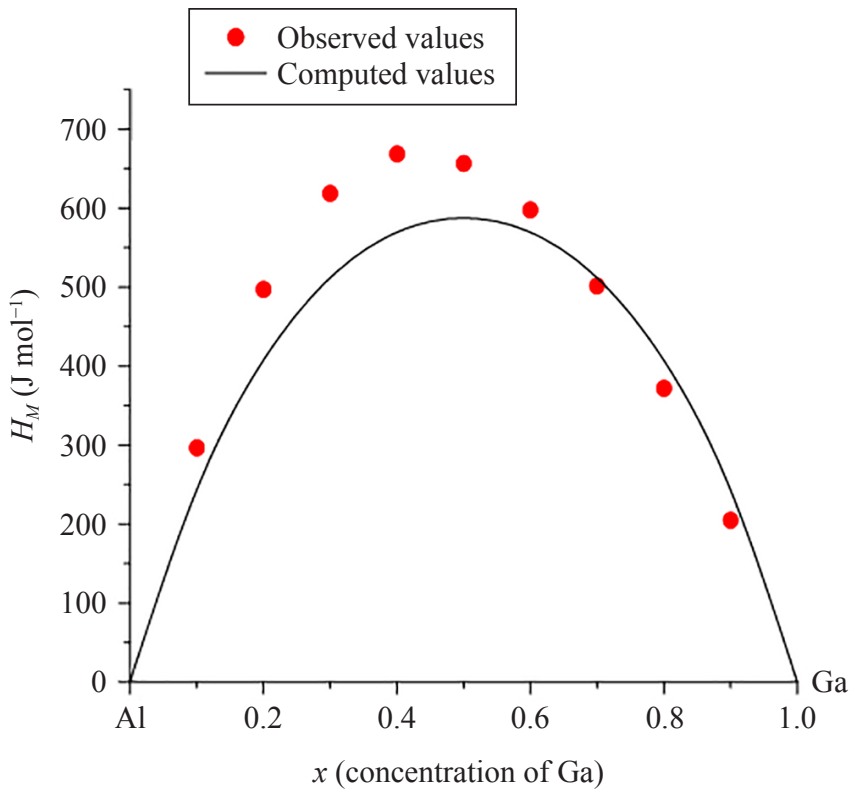

Figure 4: Heat of mixing $H_{M}$ vs. concentration of Ga of liquid Al-Ga alloy at $1023 \mathrm{~K}$.

In order to understand the ordering and segregating nature, the concentration fluctuation in the long wavelength limit $\left(S_{C C}(0)\right)$ for the Al-Ga alloy at $1023 \mathrm{~K}$ was computed using Equation 7 . The observed value of $S_{C C}(0)$ was calculated using Equation 9 and the observed value of activities of the monomers of the alloy. ${ }^{22}$ The computed, observed and ideal value of $S_{C C}(0)$ was plotted in Figure 5. There was excellent agreement between the computed and observed values. The computed and observed values of $S_{C C}(0)$ for the Al-Ga alloy at $1023 \mathrm{~K}$ were symmetric at equiatomic composition. The computed and observed values of $S_{C C}(0)$ were slightly greater than the ideal value at higher and lower concentration end of Al. At intermediate composition, the computed and observed $S_{C C}(0)$ were significantly greater than the ideal value. This result implies that the Al-Ga alloy at $1023 \mathrm{~K}$ in the liquid state is segregating at all compositions. However, the tendency of segregation of the alloy was found to be greater in intermediate compositions. The knowledge of $\alpha_{1}$ is very useful to provide immediate insight into the nature of the local arrangements of atoms in the mixture. Therefore, $\alpha_{1}$ for the Al-Ga alloy at $1023 \mathrm{~K}$ was computed using Equation 9. For binary liquid alloy, the value of $\alpha_{1}$ lies between -1 and +1 . It was observed that $\alpha_{1}$ for the Al-Ga alloy at $1023 \mathrm{~K}$ was positive throughout the whole range of concentration (Figure 6). The positive value of $\alpha_{1}$ provided the conformation of homocordination, i.e., like atoms tend to pair as the nearest neighbours in the Al-Ga alloy at $1023 \mathrm{~K}$ in the liquid state. 


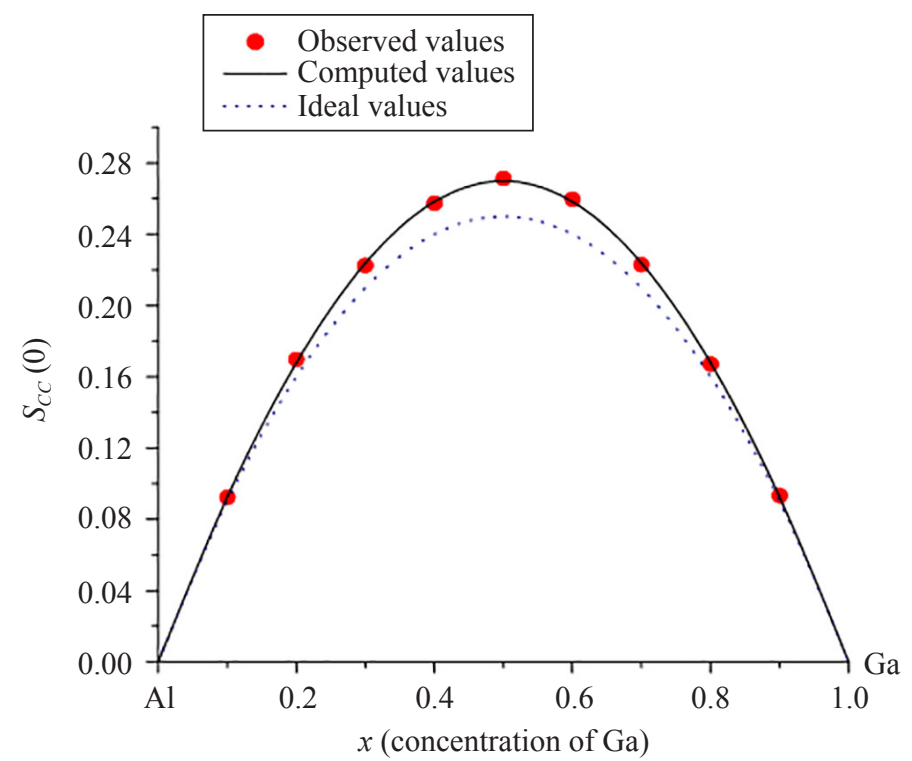

Figure 5: Concentration fluctuations in long wavelength limit $S_{c c}(0)$ vs. concentration of Ga of liquid Al-Ga alloy at $1023 \mathrm{~K}$.

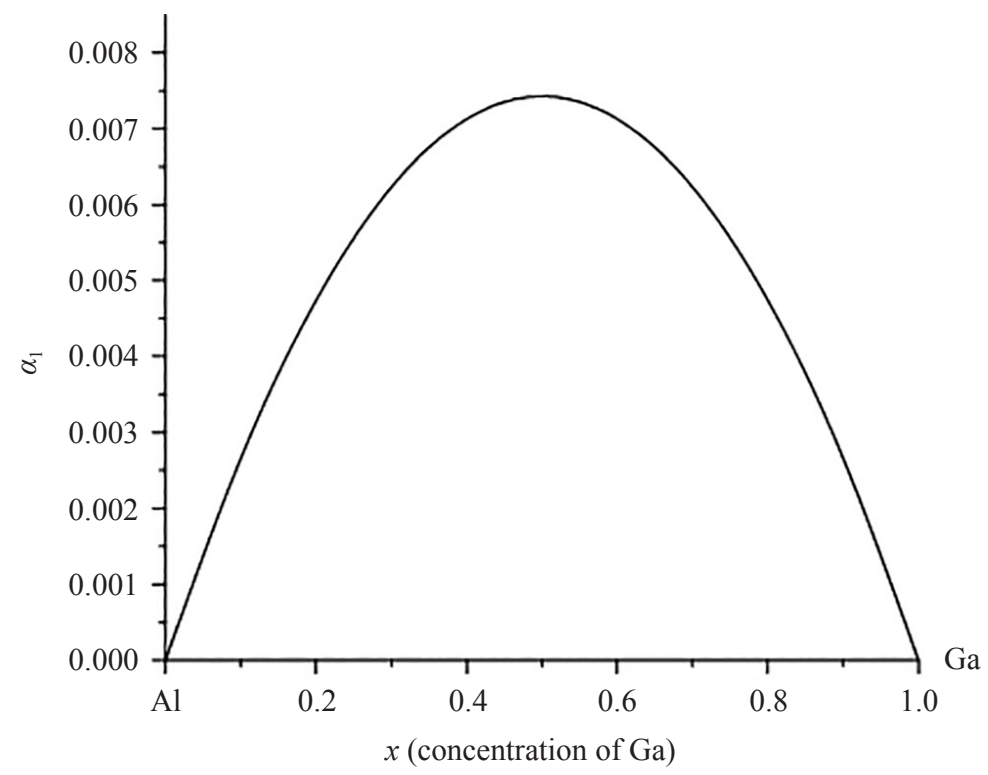

Figure 6: Short range ordering parameter $\alpha_{1}$ vs. concentration of Ga of liquid Al-Ga alloy at $1023 \mathrm{~K}$. 
The segregating and compound forming nature of an alloy can be understood in terms of the ratio of the mutual and ideal diffusion coefficients $\left(D_{M} / D_{i d}\right)$. The ratio was computed using Equation 12. This ratio for the Al-Ga alloy at $1023 \mathrm{~K}$ was found to be positive and less than unity. The nature of the curve of $D_{M} / D_{i d}$ against the concentration of Ga is upward concave (Figure 7). $D_{M} / D_{i d}$ indicates the mixing behaviour of alloy, i.e., the tendency for the compound formation and $D_{M} / D_{i d}<1$ indicates phase separation. For ideal mixing, $D_{M} / D_{i d}$ approaches 1 . Thus, the AlGa alloy is weakly segregating in nature.

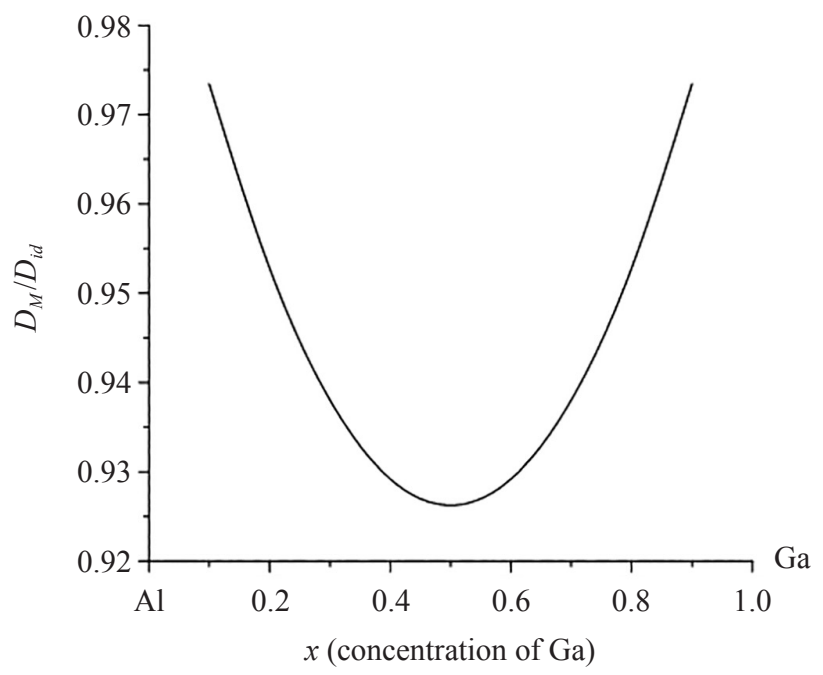

Figure 7: $D_{M} / D_{i d}$ vs. concentration of Ga of liquid Al-Ga alloy at $1023 \mathrm{~K}$.

The parameters $A_{0}$ and $A_{2}$ can be computed for higher temperature using Equation 13, assuming the variation of these parameters with temperature to be linear within a certain range of temperature. The values of these parameters at different temperatures are listed in Table 1.

Using the temperature-dependent parameters and Equation 2, the excess free energy of mixing for the Al-Ga alloy at $1100 \mathrm{~K}, 1200 \mathrm{~K}$ and $1250 \mathrm{~K}$ was computed and compared in Figure 8. The positive value of excess free energy of mixing for the Al-Ga alloy was found to decrease with a rise in the temperature. This is the usual behaviour since the excess free energy of mixing must decrease and shift towards ideal nature (ideal value of excess free energy of mixing is zero) as the temperature increases. The activities of the monomers $\mathrm{Al}$ and $\mathrm{Ga}$ of Al-Ga alloy were also computed at $1100 \mathrm{~K}, 1200 \mathrm{~K}$ and $1250 \mathrm{~K}$ using temperature dependent parameters and Equation 5 (Figure 9). It was found that the activities of the monomers shifted towards the ideality as the temperature increased. 
Table 1: Temperature dependent parameters.

\begin{tabular}{ccc}
\hline \multirow{2}{*}{ Temperature } & \multicolumn{2}{c}{ Parameter $\left(\mathrm{J} \mathrm{mol}^{-1}\right)$} \\
\cline { 2 - 3 } & \multicolumn{1}{c}{$A_{0}$} & $A_{2}$ \\
\hline $1023 \mathrm{~K}$ & 1254 & 0 \\
$1100 \mathrm{~K}$ & 1171.553 & -41.118 \\
$1200 \mathrm{~K}$ & 1064.433 & -94.518 \\
$1250 \mathrm{~K}$ & 1010.883 & -121.218 \\
\hline
\end{tabular}

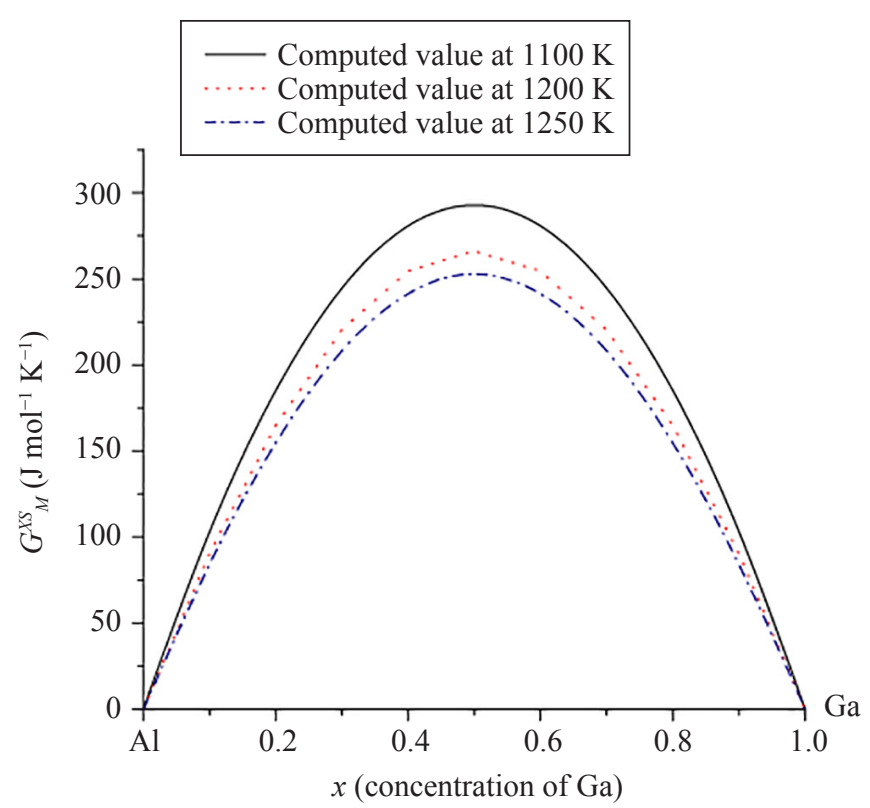

Figure 8: Excess free energy of mixing $\left(G_{M}^{X S}\right)$ vs. concentration of Ga of liquid Al-Ga alloy at $1100 \mathrm{~K}, 1200 \mathrm{~K}$ and $1250 \mathrm{~K}$.

The concentration fluctuation in the long wavelength limit for the Al-Ga alloy was also computed at $1100 \mathrm{~K}, 1200 \mathrm{~K}$ and $1250 \mathrm{~K}$ using the temperature-dependent parameters listed in Table 1 and Equation 7. The computed values of $S_{C C}(0)$ for Al-Ga alloy at different temperatures are compared in Figure 10. It was found that the $S_{C C}(0)$ goes on decreasing as the temperature increased. This clearly indicates that the behaviour of the Al-Ga alloy shifts towards the ideality as the temperature increases. Up to the temperature $1250 \mathrm{~K}, S_{C C}(0)$ varied in a usual way. However, when the temperature was increased above $1250 \mathrm{~K}, S_{C C}(0)$ became less than the ideal value at some compositions. This is unusual behaviour which is called artifact (computational inability of a model). ${ }^{23-25}$ This means the temperature dependent parameters do not vary linearly with temperature above $1250 \mathrm{~K}$. 


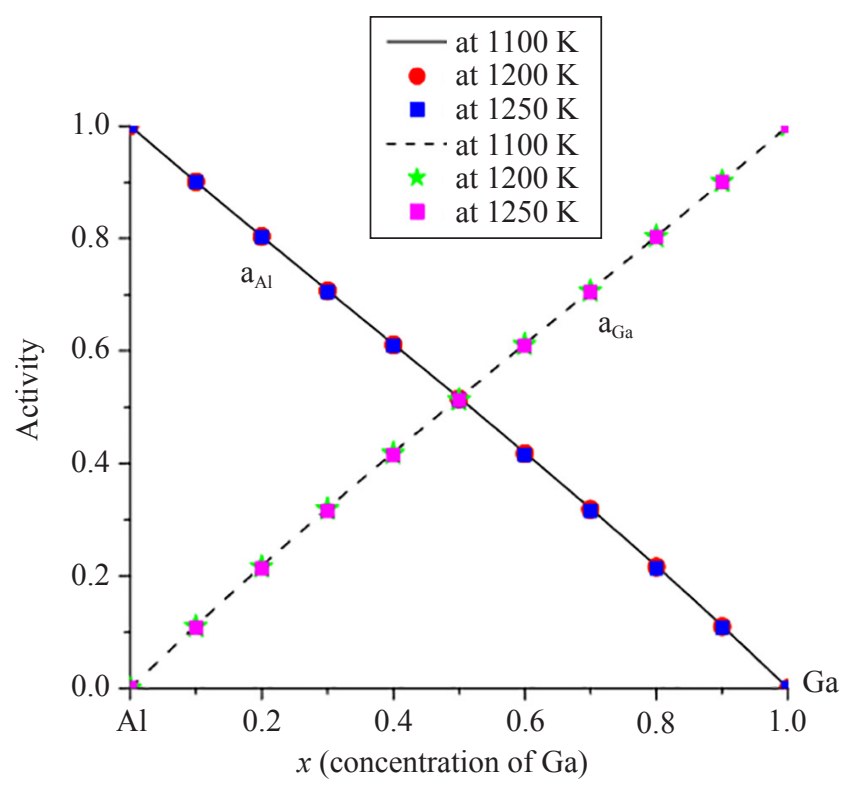

Figure 9: Activities of $\mathrm{Al}$ and $\mathrm{Ga}$ in Al-Ga alloy vs. concentration of Ga at $1100 \mathrm{~K}$, $1200 \mathrm{~K}$ and $1250 \mathrm{~K}$.

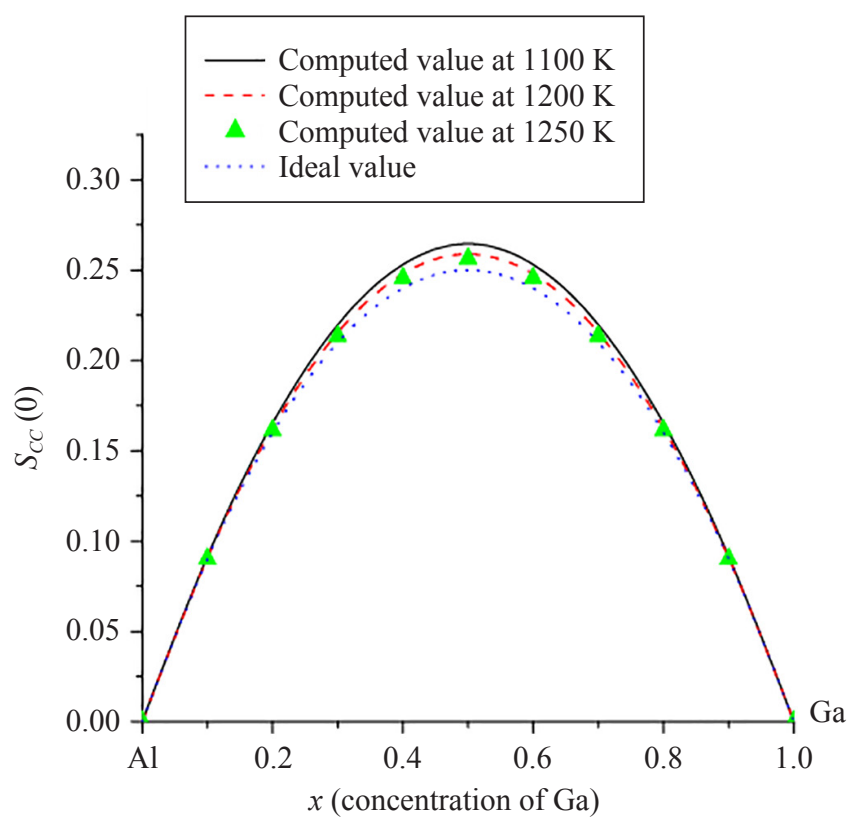

Figure 10: Concentration fluctuations in long wavelength limit $S_{c c}(0)$ vs. concentration of Ga of liquid Al-Ga alloy at $1100 \mathrm{~K}, 1200 \mathrm{~K}$ and $1250 \mathrm{~K}$. 


\section{CONCLUSION}

It is concluded from the study that the Al-Ga alloy is symmetric, weakly interacting and segregating in the liquid state. The excess free energy of mixing of the Al-Ga alloys decreases with a rise of temperature. The concentration fluctuation in the long wavelength limit of the Al-Ga alloys shifts towards the ideality with a rise in temperature.

\section{REFERENCES}

1. Flory, P. J. (1942). Thermodynamics of high polymer solutions. J. Chem. Phys., 10, 51-61. https://doi.org/10.1063/1.1723621

2. Bhatia, A. B. \& Hargrove, W. H. (1974). Concentration fluctuation and thermodynamic properties of some compound forming binary molten alloys. Phys. Rev. B., 10, 3186. https://doi.org/10.1103/PhysRevB.10.3186

3. Ruppersberg, H. \& Reiter, H. (1982). Chemical short-range order in liquid LiPb alloys. J. Phys. F Met. Phys., 12(7), 1311. https://doi.org/10.1088/0305 $-4608 / 12 / 7 / 005$

4. Hoshino, K. (1983). On the temperature dependence of the structure factor of the liquid Li-Pb alloy. Phys. F Met. Phys., 13(10), L189. https://doi.org/10.1088/03054608/13/10/001

5. Bhatia, A. B. \& Singh, R. N. (1984). A quasi-lattice theory compound forming molten alloys. Phys. Chem. Liq., 13(3), 177-190. https://doi.org/10.1080/ 00319108408080778

6. Sommer, F. (1990). Thermodynamic properties of compound-forming liquid alloys. J. Non-Cryst. Solids, 117-118(Part2), 505-512. https://doi.org/10.1016/ 0022-3093(90)90580-F

7. Singh, R. N. \& Sommer, F. (1992). Temperature dependence of the thermodynamic functions of strongly interacting liquid alloys. J. Phys. Condens. Matter., 4(24), 5345. https://doi.org/10.1088/0953-8984/4/24/004

8. Young, W. H. (1992). Structural and thermodynamic properties of NFE liquid metals and binary alloys. Rep. Prog. Phys., 55(10), 1769. https://doi.org/10.1088/ 0034-4885/55/10/003

9. Novakovic, R. (2010). Thermodynamic, surface properties and microscopic functions of liquid Al-Nb and Nb-Ti alloys. J. Non-Cryst. Solids, 356(31-32), 1593-1598. https://doi.org/10.1016/j.jnoncrysol.2010.05.055

10. Adhikari, D. et al. (2010). Structural and energetic asymmetry in liquid Ag-Al alloys. Phys. Chem. Liq., 48(6), 787-796. https://doi.org/10.1080/ 00319101003699008

11. Gohivar, R. K. et al. (2020). Artifacts in Al-Mn alloy. Phys. B Cond. Mat., 595, 412348. https://doi.org/10.1016/j.physb.2020.412348 
12. Mehta, U. et al. (2020). Prediction of thermodynamic and surface properties of ternary Ti-Si-Fe liquid alloy. Mod. Simul. Mater. Sci. Eng., 28(6), 065010. https://doi.org/10.1088/1361-651X/aba053

13. Tze, S. K. \& Reza, F. (2012). Evaluation of physical properties and casting accuracy of chrome-cobalt alloys with different casting systems and investments. J. Phys. Sci., 23, 91-102.

14. Ggenheim, E. A. (1937). Thermodynamics. Trans. Farad. Soc., 33, 151.

15. Redlich, O. \& Kister, A. (1948). Algebraic representation of thermodynamic properties and the classification of solutions. Ind. Eng. Chem., 40(2), 345-348. https://doi.org/10.1021/ie50458a036

16. Hume-Rothery, W. (1969). Atomic theory for students of metallurgy. London: The Institute of Metals.

17. Guggenheim, E. A. (1967). Thermodynamics. Amsterdam: Elsevier.

18. Warren, B. E. (1969). X-ray diffraction. Reading, MA: Addison-Wesley.

19. Cowley, J. M. (1950). An approximate theory of order in alloys. Phys. Rev., 77, 669. https://doi.org/10.1103/PhysRev.77.669

20. Singh, R. N. \& Sommer, F. (1998). Thermodynamic investigations of viscosity and diffusion of binary liquid alloys. Phys. Chem. Liq., 36(1), 17. https://doi.org/10.1080/00319109808035917

21. Darken, L. S. \& Gurry, R. W. (1953). Physical chemistry of metal. New York: McGraw Hill.

22. Hultgren. R. et al. (1973). Selected values of the thermodynamic properties of binary alloys. New York: ASM.

23. Gohivar, R. K. et al. (2020). Assessment of thermo-structural properties of $\mathrm{Al}-\mathrm{Fe}$ and Fe-Si alloys at high temperatures. Phys. Chem. Liq. https://doi.org/10.1080/00319104.2020.1793985

24. Kaptay, G. (2004). A new equation for the temperature dependence of the excess Gibbs energy of solution phases. Cal. Comput. Couple. Phase Diagr. Thermochem., 28(2), 115-124. https://doi.org/10.1016/j.calphad.2004.08.005

25. Gohivar, R. K. et al. (2020). Temperature dependence of interaction parameters of Cu-Si liquid alloy. AIP Adv., 10(085121), 1-6. https://doi.org/10.1063/5.0012834 